\title{
Diffusion of Gold Clusters on Defective Graphite Surfaces
}

\author{
Bokwon Yoon, W. D. Luedtke, Jianping Gao, and Uzi Landman* \\ School of Physics, Georgia Institute of Technology, Atlanta, Georgia 30332-0430 \\ Received: February 18, 2003; In Final Form: April 16, 2003
}

\begin{abstract}
Diffusion characteristics of gold clusters (consisting of 38, 79, 140, or 586 atoms) on two types of defective graphite surfaces, one with a one-layer deep hole and the other with a one-layer high step edge, are studied with the use of molecular dynamics simulations. The effects of the defects on the energetics of the cluster and on the diffusive trajectories are investigated. The simulations guide formulation of a simple analytic model that is demonstrated to provide an appropriate description of the energy and of the forces acting on a cluster diffusing on a graphite surface in the presence of defects.
\end{abstract}

\section{Introduction}

Explorations pertaining to the physical factors that govern the elementary processes of cluster deposition and diffusion on solid surfaces form a rapidly growing branch of experimental and theoretical research. ${ }^{1-6}$ These studies are of great relevance for elucidation of the mechanisms of thin-film growth and for the development of surface manipulation techniques.

Enhanced diffusion rates of adsorbed small metal clusters containing up to 10 atoms have been observed in early fieldion microscopy investigations ${ }^{7 \mathrm{a}}$ and described using a random walk with internal state formalism. ${ }^{7 b}$ More recently, a dislocation mechanism $^{8}$ for surface migration of large two-dimensional clusters and a collective slip-diffusion model for rapid surface diffusion of thiol-passivated gold nanoclusters on graphite ${ }^{9}$ have been proposed. Furthermore, molecular dynamics (MD) simulations revealed ${ }^{10}$ a novel surface diffusion phenomenon, where large (hundreds of atoms) three-dimensional gold clusters adsorbed on a graphite surface were predicted to undergo anomalous diffusive motion with surprisingly high rates, occurring through a collective slip-diffusion mechanism, involving long sliding trajectories, which may be described mathematically as Lévy flights. ${ }^{11}$ In that study it was also remarked that the details of the diffusion mechanism would depend on the degree of commensurability between the adsorbed cluster and the surface, on the temperature, and on the coupling strength between the adsorbate and the surface. Indeed, it had been observed experimentally that gold clusters exhibit high mobility on graphite surfaces, leading to the formation of ramified islands, ${ }^{4}$ and associated MD simulations of large Lennard-Jones clusters on a solid surface ${ }^{12}$ concluded that when the cluster is incommensurate with the substrate, fast diffusion occurs via a Brownian-like mechanism.

Although material surfaces encountered in experimental work commonly contain various types of defects, such as steps, holes or pits, interstitials and bumps, ${ }^{13-16}$ most theoretical studies of cluster surface diffusion processes have been performed for ideal surfaces. Consequently, we focus in this paper on MD simulations pertaining to the interaction between gold nanoclusters and a number of defects commonly observed on graphite surfaces; specifically, we study cluster-hole and cluster-step interactions.

The molecular dynamics simulation methodology, interatomic interaction potentials, and simulation procedure are described in section 2. Results for the energetics and diffusion characteristics of gold clusters on defective graphite surfaces obtained through MD simulations, as well as via a formulated analytic model, are given in section 3 . We summarize our results in section 4 .

\section{Simulation Method}

In our MD simulations we employ the many-body embeddedatom potentials ${ }^{17}$ for the interaction between the gold atoms, and a 6-12 Lennard-Jones (LJ) potential for the interaction between gold and graphite atoms; ${ }^{10}$ the LJ parameters are $\epsilon(\mathrm{Au}-\mathrm{C})=0.01273 \mathrm{eV}$ and $\sigma(\mathrm{Au}-\mathrm{C})=2.9943 \AA$ with a cutoff distance of $2.5 \sigma(\mathrm{Au}-\mathrm{C})$. The gold nanocrystals have a facecentered-cubic structure with a truncated octahedral (TO) morphology. In this study we treat the graphite surface atoms as static, because previous simulations of cluster diffusion on graphite have indicated an insensitivity of the results to the dynamics of the graphite atoms (i.e., similar results were obtained when treating the graphite surface atoms statically or dynamically ${ }^{9}$ ). At this juncture several issues may be raised: (i) The interaction between atoms of the gold cluster and lowcoordinated carbon atoms (found on defective surfaces) may differ from that with carbon atoms of perfect graphite; in particular, the interaction may be influenced by dangling bonds associated with the defects and/or modified by structural reconstructions involving low-coordinated defective graphite sites. (ii) A sufficiently strong interaction between the cluster and the defective graphite may lead to deformations of the surface (particularly in the vicinity of the defect). Nevertheless, treating the graphite surface statically, may serve as a valuable starting point for further studies of the interaction of metal clusters with defective graphite surfaces.

In these investigations two MD simulation methods were used: (i) Microcanonical (i.e., constant-energy) simulations were employed for long (240 ns) simulations of freely diffusing clusters. These simulations allow direct observation of the general behavior and trends in the diffusion of clusters of various sizes and at different temperatures, as they interact with graphite surface defects. (ii) In some cases, we used constrained MD simulations, where the separation between the cluster center-of mass (CM) and a surface defect is held constant while all other degrees of freedom of the cluster are allowed to vary. By performing such simulations for a wide range of cluster-defect 

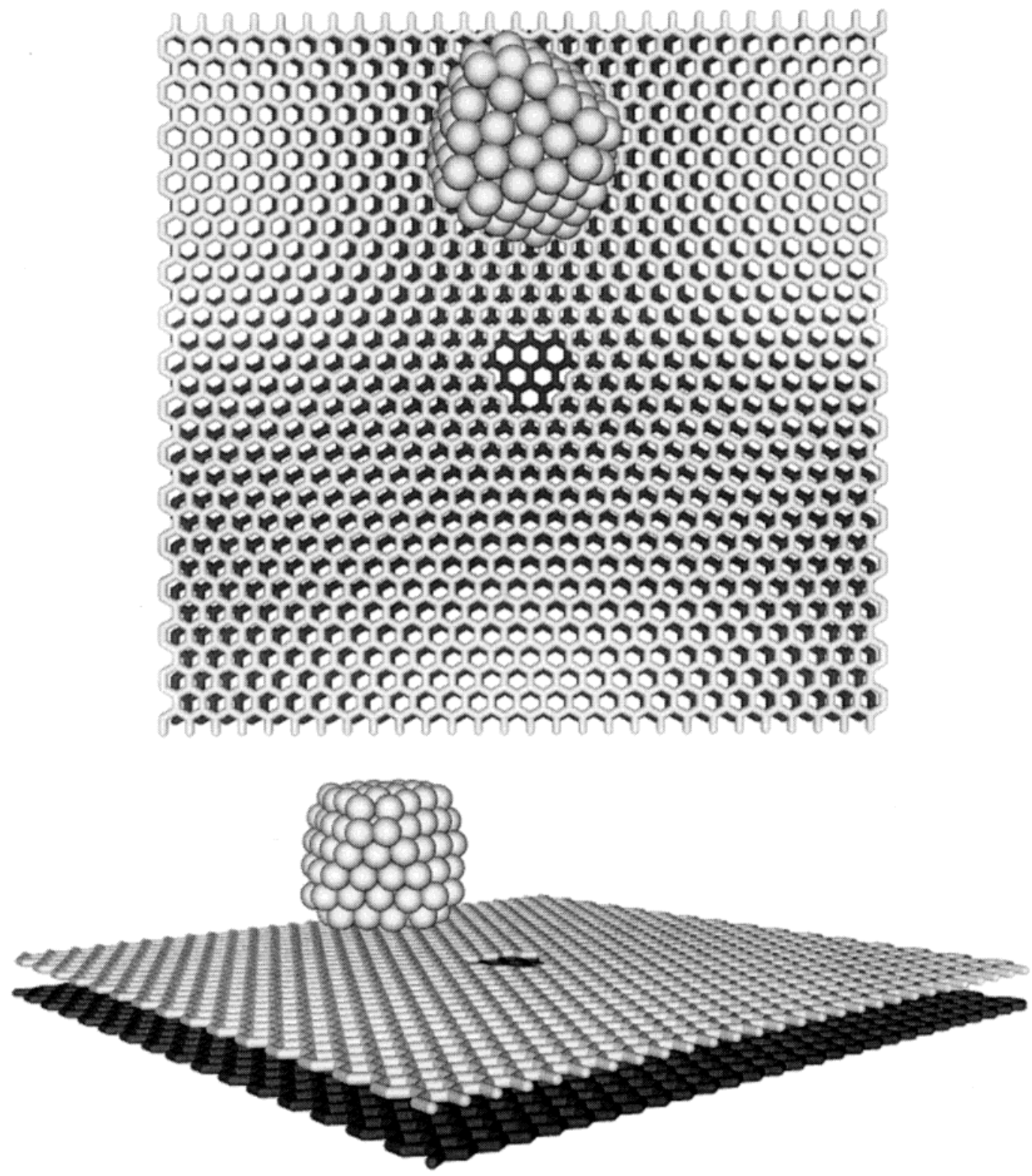

Figure 1. Top view (upper) and side view (bottom) of an $\mathrm{Au}_{140}$ cluster adsorbed on a graphite surface with a 13-atom hole defect at the center of the top graphite sheet.

separations, one can deduce the forces and energies required for a cluster to either escape, in some cases, from a defect or, under other circumstances, approach and even traverse a surface defect. These types of studies can serve as important guides in constructing simple models to describe cluster-defect interactions.

The graphite substrate was modeled by a slab (see below) with periodic boundary conditions (PBC) imposed in the two directions defining the basal plane; the dimensions of the surface were taken large enough to avoid influence of the PBC on the results. The equations of motion were integrated using the velocity Verlet algorithm with a time-step of $3 \mathrm{fs}$; with this time step the energy of the system was found to remain essentially constant even for very long simulations (e.g., $240 \mathrm{~ns})$.

\section{Results}

3.1. Graphite Surface with a Hole. A graphite surface with a hole is modeled by an upper graphite sheet with 13 atomic vacancies at its center, positioned on top of a supporting defect- free lower graphite sheet that is separated from the top sheet by the appropriate layer spacing $(3.35 \AA)$ in the $(0001)$ direction (Figure 1). Unless otherwise specified, our simulations were performed at $300 \mathrm{~K}$, and the adsorbed gold cluster was positioned initially with one of its (111) facets contacting the graphite surface. Initially, the cluster is placed on a defect-free region of the surface and it is thermally equilibrated to the desired temperature. The dimensions of the graphite surface are $68.87 \AA$ by $68.16 \AA$, and with the PBC one hole on the surface corresponds to a hole density of $2.13 \times 10^{12} \mathrm{~cm}^{-2}$. Figure 2 displays trajectories of the center of mass (CM) for two gold clusters, $\mathrm{Au}_{140}$ and $\mathrm{Au}_{586}$, recorded during $240 \mathrm{~ns}$ of constant energy MD simulations. The centers of the holes are shown as open circles. The trajectories of the clusters exhibit patterns similar to those simulated previously on a defect-free graphite $^{9,10,18}$ A new aspect that we find here, however, is the formation of an extended excluded zone for cluster diffusion in the region surrounding the defect hole. Namely, the clusters experience repulsion from the holes, as reflected in the trajectories shown in Figure 2. The diffusion constants of the 

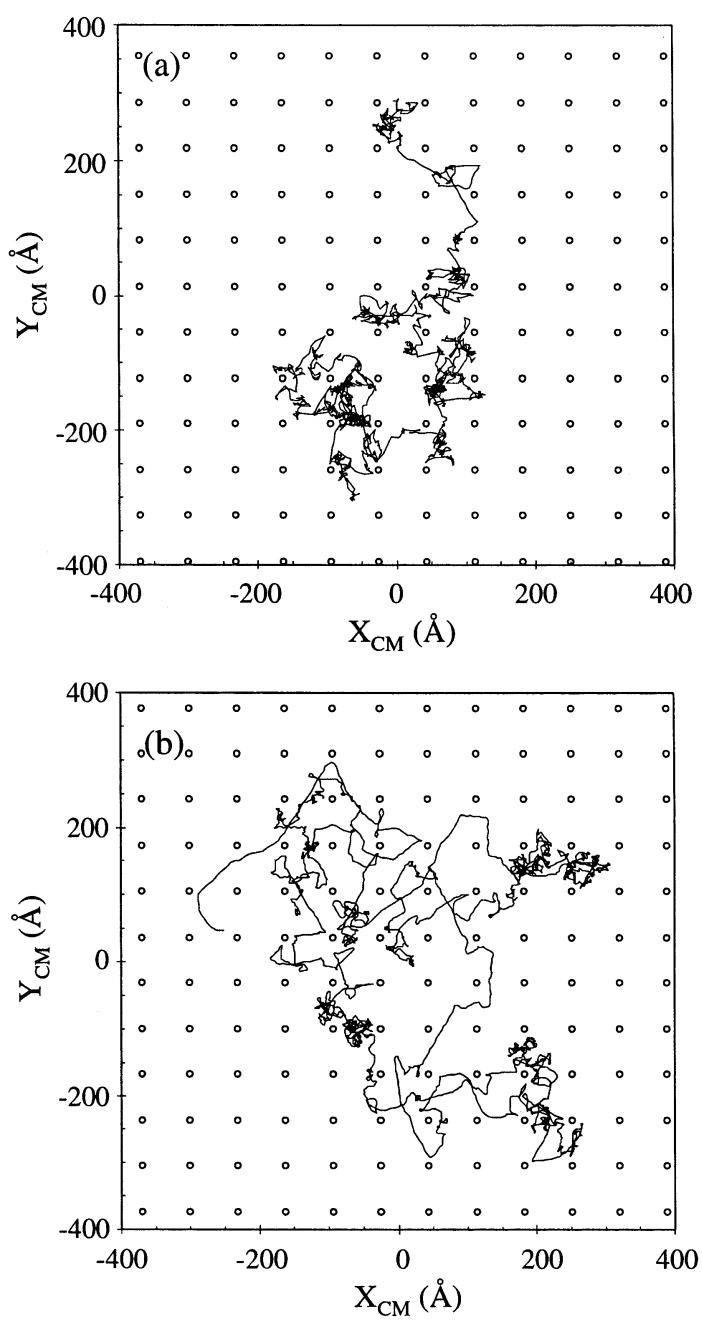

Figure 2. CM (center-of-mass) trajectories of gold clusters on a graphite surface with a hole: (a) $\mathrm{Au}_{140}$ cluster and (b) $\mathrm{Au}_{586}$ cluster. The trajectories were recorded in molecular dynamics simulations at $300 \mathrm{~K}$. In this figure the computational cell, whose planar dimensions are $68.87 \AA$ by $68.16 \AA$, has been replicated. Open circles represent only the positions of the defect holes, but not their size.

two clusters, determined from the relationship $D=\left\langle\left[\mathbf{R}_{\mathrm{CM}}(t)-\right.\right.$ $\left.\left.\mathbf{R}_{\mathrm{CM}}(0)\right]^{2}\right\rangle / 4 t$ with $t=12 \mathrm{~ns}$, are $1.49 \times 10^{-5} \mathrm{~cm}^{2} / \mathrm{s}$ for $\mathrm{Au}_{140}$ and $4.24 \times 10^{-5} \mathrm{~cm}^{2} / \mathrm{s}$ for $\mathrm{Au}_{586}$. These values are $30 \%$ and $10 \%$ smaller than the diffusion constants measured in the simulations of gold clusters on a defect-free graphite surface. ${ }^{18}$

As indicated above normal diffusion is characterized by a variance of the displacements $\sigma^{2}(t)=\left\langle[\mathbf{R}(t)-\mathbf{R}(0)]^{2}\right\rangle \sim t^{\gamma}$ growing in the long-time limit linearly with $t$, i.e., $\gamma=1$. Superdiffusion $(\gamma>1)$ may occur when the trajectories exhibit long displacements (flights), termed "Lévy flights" ${ }^{11}$ characterized by power-law (rather than exponential) flight-time $t_{\mathrm{F}}$ probability distribution functions, $P\left(t_{\mathrm{F}}\right) \sim t_{\mathrm{F}}{ }^{-\mu}$ with $1<\mu<3$; for such processes the mean square displacement is divergent with time. Analysis reveals that the cluster CM trajectories recorded in our simulations may be divided into "free flight" trajectories (with associated free-flight time intervals, $t_{\mathrm{F}}$ ) and "sticking" trajectories (with associated sticking time intervals $t_{\mathrm{S}}$, and a corresponding sticking-time distribution $P\left(t_{\mathrm{S}}\right) \sim t_{\mathrm{S}}{ }^{-v}$ ). For the $\mathrm{Au}_{140}$ cluster diffusing on the graphite surface in the presence of the hole defect, we find $\mu=2.5 \pm 0.4$ and $v=$ $2.8 \pm 0.5$, indicating Levy-flight character of the diffusive motion. However, for the exponent $\gamma$ of the mean-square CM displacements we find a mean value $\gamma=1$, implying normal diffusion. ${ }^{19}$
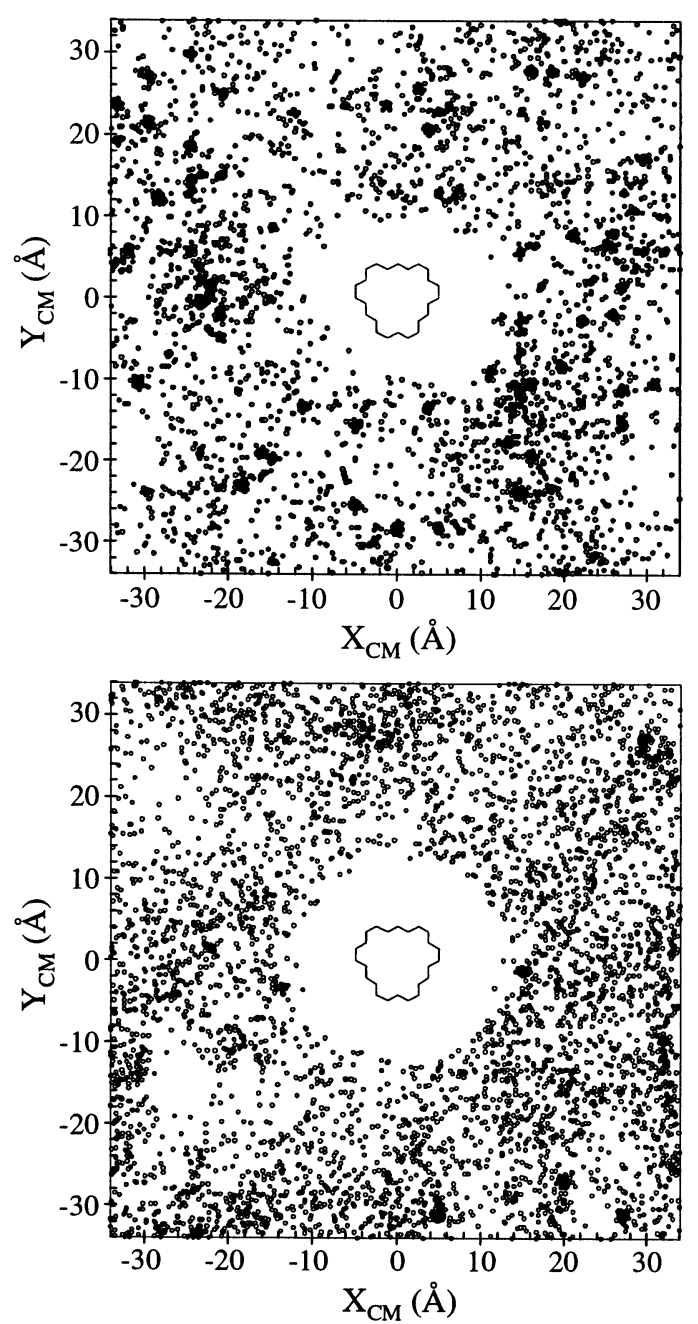

Figure 3. CM positions sampled for every 48 ps recorded during 240 ns MD simulations: (a) the $\mathrm{Au}_{140}$ cluster and (b) the $\mathrm{Au}_{586}$ cluster. Periodic boundary conditions are used and the dimensions of the surface computational cell are as given in the figures. The polygons at the centers of the figures depict the edge atoms constituting the hole. The hole is seen to be surrounded by an excluded zone for clusters with a radius roughly equal to the hole radius plus the cluster radius. When the cluster is in a sticking state, the CM positions accumulate in the local region around the sticking position, resulting in a darkened region in the figure.

To better exhibit the aforementioned excluded zone for cluster diffusion, we sample the cluster's CM position at $48 \mathrm{ps}$ intervals during a $240 \mathrm{~ns}$ simulation and plot them cumulatively on the graphite surface (Figure 3). The excluded zones are larger than the hole area (displayed by polygons of edge atoms) by approximately the radius of the contact area of the cluster with the underlying surface. Darkened regions in the figure are formed by the accumulation of $\mathrm{CM}$ positions in a small region in which the cluster exhibits (for a certain time interval) oscillatory vibrational motions about a sticking (pinning) site. The alternation between local oscillatory "sticking" states and free diffusive motion is known as "stick-slip" diffusion, 9,10 and it may exhibit rich behavior that is related to Levy flights. ${ }^{11}$

To further explore the influence of the defect hole on the diffusive motion of the cluster, we perform constrained MD simulations. The constraint that we impose consists of maintaining a constant distance $R_{\mathrm{CM}}$ between the center of the hole and the cluster CM. As mentioned earlier, initially the cluster is thermally equilibrated at $300 \mathrm{~K}$ on a defect-free region of the surface. To average over many cluster-surface bonding ar- 

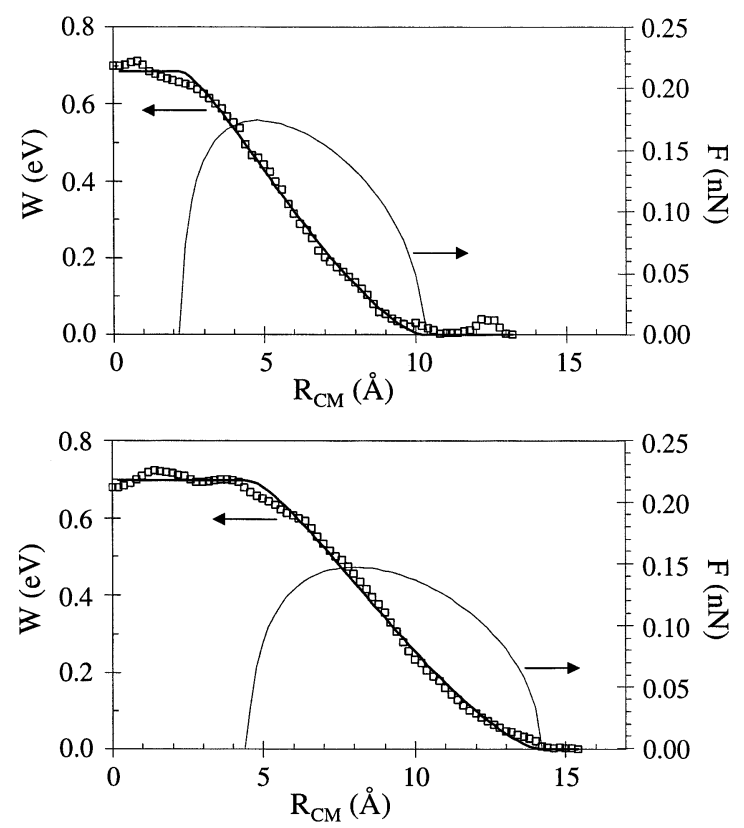

Figure 4. Work $W=-\int_{R_{\mathrm{CM}}\left(R_{\mathrm{r}}\right.}^{R_{\mathrm{r}}}\langle\mathrm{d} r$ calculated using $300 \mathrm{~K}$ constrained MD simulations for (a) an $\mathrm{Au}_{140}$ cluster and (b) an $\mathrm{Au}_{586}$ cluster. Also shown are the energy (thick solid curve) and the force (thin solid curve) calculated from the COM (see text).

rangements consistent with a fixed $R_{\mathrm{CM}}$, the cluster is allowed to move freely in the directions perpendicular to the vector $\mathbf{R}_{\mathrm{CM}}$ that connects the center of the hole to the cluster's CM. This is done for a range of $R_{\mathrm{CM}}$ values starting from nonoverlap of the cluster and hole, to partial overlap, and finally to full coverage of the hole by the cluster. For a given distance $R_{\mathrm{CM}}$, we average the radial force component $\left\langle f_{\mathrm{r}}\right\rangle$, parallel to $\mathbf{R}_{\mathrm{CM}}$, acting on the cluster over times of 1.2-2.4 ns. Profiles of the work as a function of $R_{\mathrm{CM}}$, displayed by open rectangles in Figure 4 , are obtained by integrating $\left\langle f_{\mathrm{r}}\right\rangle$ from a sufficiently long distance from the hole,

$$
W\left(R_{\mathrm{CM}}\right)=-\int_{R_{\mathrm{CM}}^{(0)}}^{R_{\mathrm{CM}}}\left\langle f_{\mathrm{r}}\right\rangle \mathrm{d} r
$$

and it represents the energy necessary to bring a cluster to a distance $R_{\mathrm{CM}}$ from the hole starting from a remote position. Because we average the radial component of the force over many cluster-surface configurations, $W\left(R_{\mathrm{CM}}\right)$ includes entropic contributions. The maximum value of the work represents the energy barrier for the cluster to completely cover the hole. The measured energy barrier is about $0.7 \mathrm{eV}$ for both $\mathrm{Au}_{140}$ and $\mathrm{Au}_{586}$ - the insensitivity to the size of the cluster originates from the fact that energy changes are related solely to the degree of coverage of the hole by a cluster, and thus once the hole is fully covered, the energy cannot increase further and it is the same for all clusters capable of fully covering the hole.

To model the $W\left(R_{\mathrm{CM}}\right)$ work (energy) curves, we employ a "cluster overlap" model (COM). We approximate the lateral hole geometry and the cluster-surface contact region as disks of radii $r$ and $R$, respectively. When the cluster is distant and it does not overlap the step edge of the hole, the cluster-surface interaction energy may be written as $E_{0}=-\epsilon_{0} A_{\mathrm{C}}$, where $\epsilon_{0}$ is the (positive) interaction energy (per unit area) between graphite and a $\mathrm{Au}[111]$ surface and $A_{\mathrm{C}}=\pi R^{2}$ is an estimate of the cluster-surface contact area in which the contact area of the cluster is modeled as a disk of radius $R$. The interaction between the cluster and the defect starts to have an effect when the cluster periphery begins to overlap the step edge at the periphery of

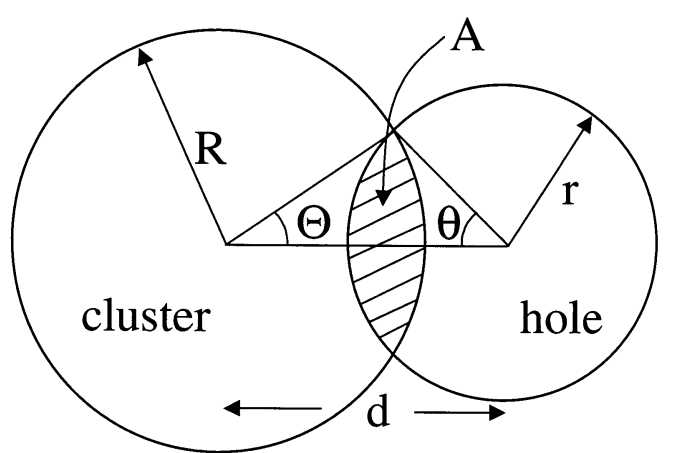

Figure 5. Geometrical schematic of the cluster-overlap model (COM). The cluster and the hole are described by two-dimensional disks of radius $R$ and $r$, respectively. The lens-shaped overlap area $A$ of the cluster with the hole is depicted by the striped region.

the hole. When such overlap occurs, the overall interaction energy between the cluster and the surface is reduced due to the diminished cluster-surface contact area; in our model, only the contribution from interactions between the cluster and the topmost graphite layer is influenced by the hole defect, whereas the remaining contribution to the energy coming from the interaction between the cluster and the second graphite layer (that is the layer underneath the topmost layer) is unaffected. Denoting by $A$ the area of the cluster-hole overlap (Figure 5) and by $\epsilon_{1}$ the interaction energy (per unit area) between an $\mathrm{Au}(111)$ gold surface and the second (and lower) graphite layer, the cluster-hole overlap energy in our model may be written as the difference between the overlapping energy, $E_{1}=$ $-\epsilon_{0}\left(A_{\mathrm{C}}-A\right)-\epsilon_{1} A$, and the energy associated with complete nonoverlap between the cluster and the hole defect $\left(E_{0}\right)$. This gives $E=E_{1}-E_{0}=\left(\epsilon_{0}-\epsilon_{1}\right) A$, representing the net increase in energy due to the cluster-hole overlap. Defining the positive energy difference $\epsilon=\epsilon_{0}-\epsilon_{1}$ as an effective cluster-hole overlap energy, we obtain $E=\epsilon A$. Setting $d=$ cluster-hole CM separation (Figure 5) yields

$E= \begin{cases}0 & \text { for } d>r+R \text { (no overlap) } \\ \epsilon A & \text { for } R+r>d>R-r \text { (partial overlap) } \\ \epsilon A_{\mathrm{H}} & \text { for } d>R-r(\text { complete overlap and maximum } \\ & \left.\text { energy, } A_{\mathrm{H}}=\pi r^{2}\right)\end{cases}$

Expressing the overlap area $\mathrm{A}$ in terms of the geometrical parameters of the model, the energy $E$ may be written as

$$
\begin{aligned}
E & =\epsilon A \\
& =\epsilon\left[r^{2} \theta+R^{2} \Theta-\right. \\
& \left.\frac{1}{2} \sqrt{(-d+r+R)(d+r-R)(d-r+R)(d+r+R)}\right]
\end{aligned}
$$

where $\theta$ and $\Theta$, depicted in Figure 5, can be expressed as functions of $r, R$, and $d$

$$
\begin{aligned}
& \theta=\cos ^{-1}\left(\frac{d^{2}+r^{2}-R^{2}}{2 d r}\right) \\
& \Theta=\cos ^{-1}\left(\frac{d^{2}+R^{2}-r^{2}}{2 d r}\right)
\end{aligned}
$$

The solid curves in Figure 4 are obtained through fitting the work profiles with eq 3 , and they represent effective clusterhole overlap energies. These overlap energies lead to repulsive forces $F=-\partial_{d} E(d)$ acting on the cluster. If the cluster has 

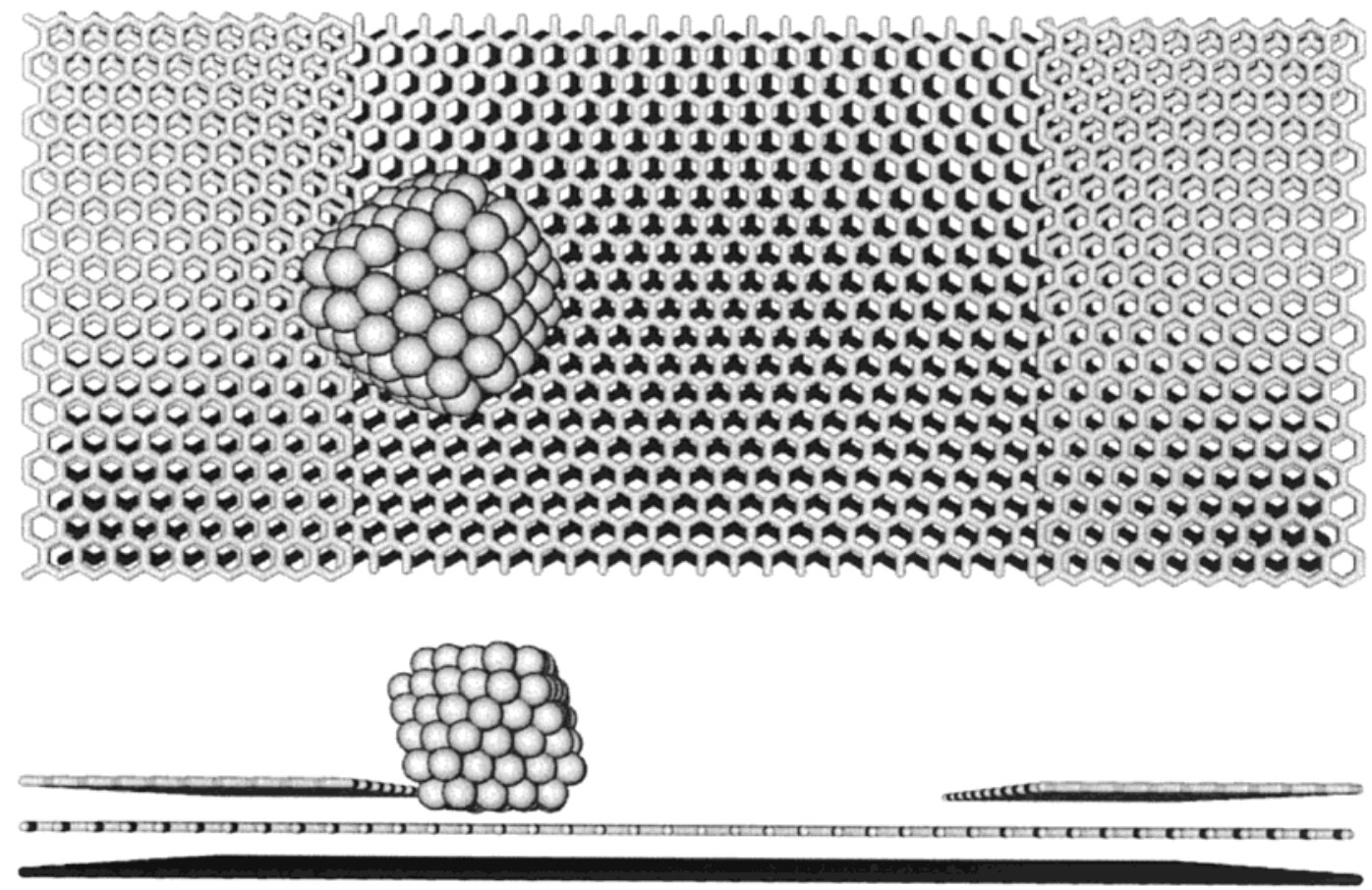

Figure 6. Top view (upper) and side view (bottom) of an $\mathrm{Au}_{140}$ cluster adsorbed on a graphite surface with ascending steps. The cluster is located on the lower terrace.

TABLE 1: Values of $r, R$, and $\epsilon$, and the Maximum Values of the Repulsive Radial Force Obtained from a Least-Squares Fit with the Use of $\mathrm{Eq}^{a}$

\begin{tabular}{lcccc}
\hline & $r(\AA)$ & $R(\AA)$ & $\epsilon\left(\mathrm{eV} / \AA^{2}\right)$ & $F_{\max }(\mathrm{nN})$ \\
\hline $\mathrm{Au}_{140}$ & 4.00 & 6.26 & 0.0136 & 0.174 \\
$\mathrm{Au}_{586}$ & 4.83 & 9.37 & 0.0095 & 0.147
\end{tabular}

${ }^{a}$ Results are given for $\mathrm{Au}_{140}$ and $\mathrm{Au}_{586}$ clusters interacting with a 13-atom hole defect on the top layer of a graphite surface.

sufficient translational kinetic energy it may overcome the energy barrier (associated with complete hole coverage in the COM) and pass over the hole. Note that the average translational kinetic energy of a cluster at $300 \mathrm{~K}$ is $\sim 0.04 \mathrm{eV}$, which is much smaller than the calculated energy barrier of about $0.7 \mathrm{eV}$ needed to traverse the hole. The values of $r, R$, and $\epsilon$, and the maximum values of the radial repulsive force are presented in Table 1. Though the actual shapes of the hole and the cluster's contact face may deviate somewhat from being circular, the COM model fits well to the simulation results and the values of the model parameters obtained through such fit are physically reasonable.

The cluster-hole repulsive force $F=-\partial_{d} E(d)$ obtained from eqs 3 and 4 is given by

$$
F=\frac{\epsilon}{d} \sqrt{(-d+r+R)(d+r-R)(d-r+R)(d+r+R)}
$$

within the regime $R+r>d>R-r$ (partial overlap), and it vanishes otherwise. The force achieves its maximum value $F_{\max }=2 \epsilon r$ at $d=\sqrt{R^{2}-r^{2}}$ and is independent of the cluster size. As shown in Table 1, the difference between the maximum forces calculated for $\mathrm{Au}_{140}$ and $\mathrm{Au}_{586}$ is $\sim 20 \%$.

3.2. Graphite Surface with Linear Ascending Steps. Straight step edges are another common type of surface feature that can significantly influence the surface diffusion of clusters. To extend our study to this case, we again perform constant energy and constrained MD simulations of gold clusters on a graphite surface with a one-layer high step and focus initially on the case where a cluster is on the lower terrace of the step (Figure 6).

As a first study aimed at gaining information about the influence of surface steps on cluster diffusion, $\mathrm{Au}_{38}$ and $\mathrm{Au}_{140}$ clusters, positioned on the lower side of the step, are thermally equilibrated to $300 \mathrm{~K}$ and then allowed to diffuse freely on the surface (at constant energy). Figure 7 shows the trajectories and lateral displacements (parallel to the step) of these clusters over a time period of $240 \mathrm{~ns}$. Both clusters spend most of the simulation time at the step. Only two escape events were observed during the $240 \mathrm{~ns}$ interval for the $\mathrm{Au}_{38}$ cluster (Figure $7 \mathrm{a})$, and no escape event occurred for the larger $\mathrm{Au}_{140}$ cluster (Figure 7b). However, the mobility along the step is surprisingly high, especially for $\mathrm{Au}_{140}$. Diffusion constants calculated from the simulations using $D^{(\text {step })}=\left\langle\left[Y_{\mathrm{CM}}(t)-Y_{\mathrm{CM}}(0)\right]^{2}\right\rangle / 2 t$, are $1.23 \times 10^{-5} \mathrm{~cm}^{2} / \mathrm{s}$ for $\mathrm{Au}_{38}$ and $9.25 \times 10^{-4} \mathrm{~cm}^{2} / \mathrm{s}$ for $\mathrm{Au}_{140}$.

To increase the probability for a cluster to escape from the step, we performed for two cluster sizes simulations at higher temperatures (see Figure 8). As the temperature increases, the escape probability increases in both cases. For the larger clusters, higher temperatures are required to obtain comparable escape probabilities. The simulations at higher temperature are also accompanied by structural changes of the clusters (see Figure 9).

Similar to our study of cluster-hole overlap, we performed constrained MD simulations at $300 \mathrm{~K}$, allowing calculation of the forces acting on the cluster which can be used in the evaluation of the corresponding work profiles (energy changes as a function of the distance between the cluster and the step)we remark that though these simulations allow us to explore the energetics of the cluster-step interactions in a well-defined manner, they do not necessarily yield the physical step-climbing mechanism because of the imposed constraint. The constraint consists of holding constant the distance between the step and the cluster's CM, while allowing the cluster to diffuse freely parallel to the step (the $y$-direction). The position of the step is taken as the origin, $X_{\mathrm{CM}}=0$, and the above constrained 

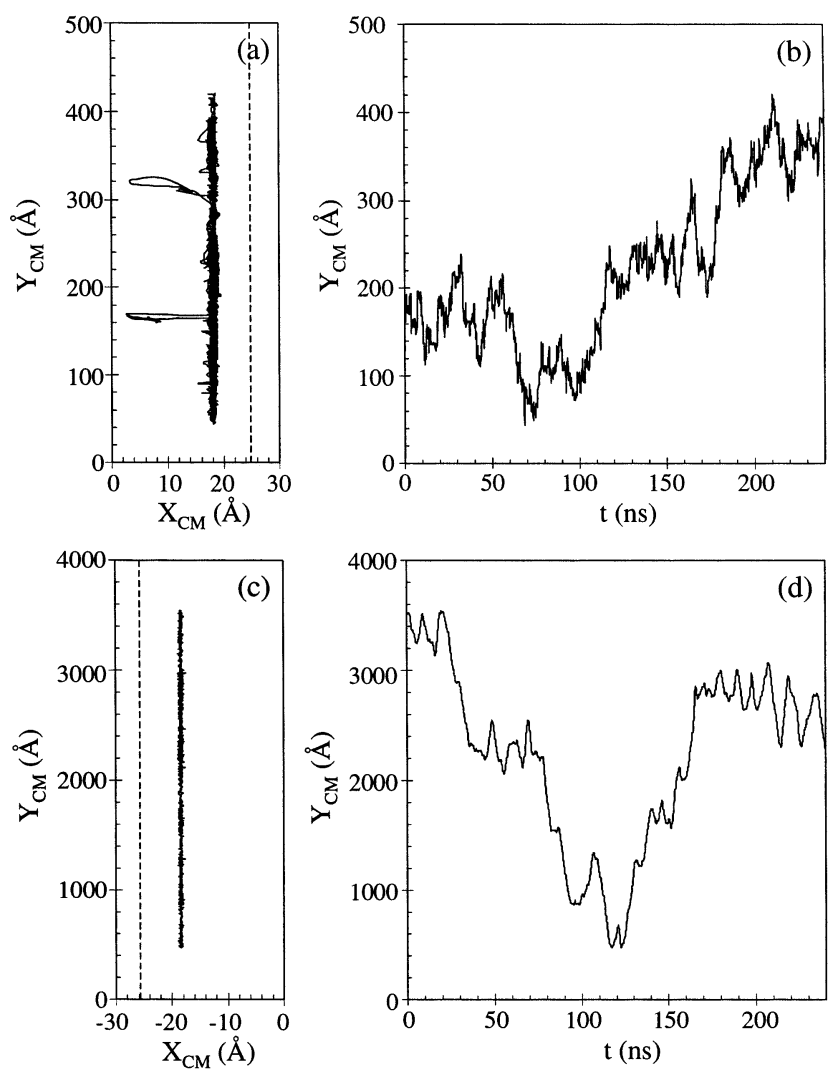

Figure 7. Trajectories of gold clusters diffusing at $300 \mathrm{~K}$ located on the lower terrace of a graphite surface and interacting with an ascending step (dashed line). The trajectories in (a) and (b) are for an $\mathrm{Au}_{38}$ cluster, and those in (c) and (d) are for an $\mathrm{Au}_{140}$. cluster. The total time span for both MD simulations is $240 \mathrm{~ns}$. Note the different length scales for diffusion parallel to the step ( $Y$ direction).

simulations are performed for various values of $X_{\mathrm{CM}}$ away from the step. Analogous to our previous study, we measure the average $x$-component $\left\langle f_{x}\right\rangle$ of the force acting on the cluster and integrate it from a point $X_{\mathrm{CM}}^{(0)}$ far from the step edge to $X_{\mathrm{CM}}$

$$
W\left(X_{\mathrm{CM}}\right)=-\int_{X_{\mathrm{CM}}^{(0)}}^{X_{\mathrm{CM}}}\left\langle f_{x}\right\rangle \mathrm{d} x
$$

The work profiles displayed in Figure 10 for $\mathrm{Au}_{38}$ and $\mathrm{Au}_{140}$ represent energy changes due to the cluster-step interactions. As evident from the snapshots of the cluster shown in the figure, the $\mathrm{Au}_{38}$ cluster undergoes structural changes at different stages of the constrained MD simulations. The main structural change is associated with the climbing process by the cluster over the step edge, i.e., when the work reaches the maximum value. We note that the structural change was only found for the smaller cluster, whereas the larger $\mathrm{Au}_{140}$ cluster maintained its TO motif throughout the entire constrained simulation.

The development of a model for the cluster-step interaction is a significantly more complex task than the cluster-hole case which we discussed earlier (see section 3.1). Consequently, we limit ourselves here to some general observations about the cluster-step interaction, which may be considered in three regimes:

(i) When a cluster resides on, and is in full contact with, the lower terrace (see Figure 10), energy changes (associated with motion of the cluster on the surface) are due to the interaction between the cluster and the linear step edge itself, i.e., the terminal end of a graphite sheet. As seen in Figure 10, the minima in the cluster-step energy curves for $\mathrm{Au}_{38}$ and $\mathrm{Au}_{140}$ at $300 \mathrm{~K}$ are in this regime ca. -0.2 and $-0.5 \mathrm{eV}$, respectively.
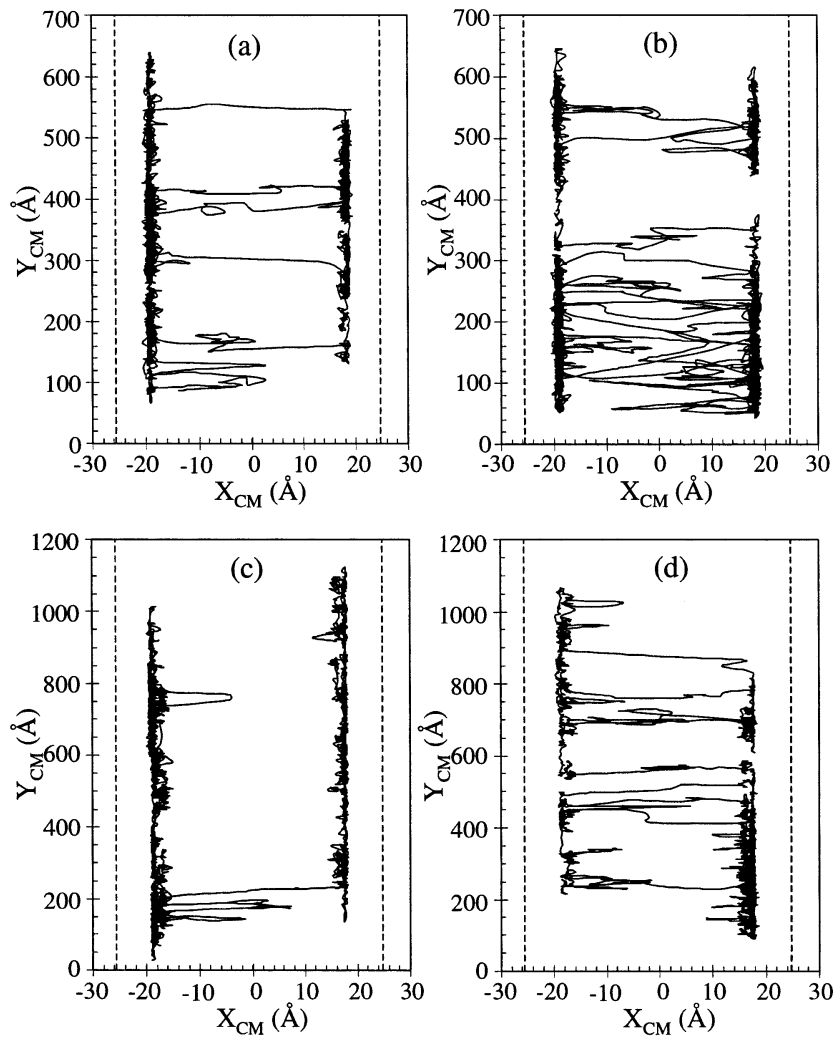

Figure 8. Trajectories of gold clusters located on a lower terrace of a graphite surface and interacting with two bounding ascending steps (dashed lines): (a) $\mathrm{Au}_{38}$ at $350 \mathrm{~K}$, (b) $\mathrm{Au}_{38}$ at $400 \mathrm{~K}$, (c) $\mathrm{Au}_{79}$ at 450 $\mathrm{K}$, and (d) $\mathrm{Au}_{79}$ at $500 \mathrm{~K}$. (a), (b), and (c) are from a $240 \mathrm{~ns} \mathrm{MD}$ simulation, and (d) is from a 120 ns simulation.

Because the average cluster translational kinetic energies at 300 $\mathrm{K}$ are of the order of $\sim 0.04 \mathrm{eV}$, we conclude that events where the cluster "escapes" from the vicinity of the step will occur only rarely, which is in line with the simulation results. One expects that the escape probability will increase at higher temperatures and that the escape rates will decrease for larger clusters, which is again consistent with our earlier observations.

(ii) When the cluster begins to lose full contact with the lower terrace and moves upward, it is in an intermediate state where it is more difficult to discern and model simply the origins of energy and force variations. One observes gradual tilting and rotational motion of the cluster in this transitional climbing state (note the $\mathrm{Au}_{140}$ cluster in Figure 10). We remark that the maximum in the cluster-step energy (the energy barrier for passing over the step from the lower terrace) corresponds to the cluster in this transitional stage.

(iii) When the cluster reaches the top terrace such that part of its contact area resides on the top surface and the other part "hangs over" the step, the situation is very similar to the clusterhole case discussed by us previously, where now the radius of the "hole" is effectively infinite. As a result, during this stage, ideas developed in the context of the cluster-hole interaction may be used. Once the cluster has reached this stage, and even slightly before, it experiences repulsive forces driving it away from the step and lower terrace and further into the upper terrace region.

3.3. Graphite Surface with Linear Descending Steps. Our discussion in this section focuses on the energetics and diffusion characteristics of a cluster residing initially on the upper terrace of a step, examining the influence of the descending step on the behavior of the cluster (see Figure 11 for a snapshot from the simulation of the diffusion of an $\mathrm{Au}_{140}$ cluster on an upper 
(a)
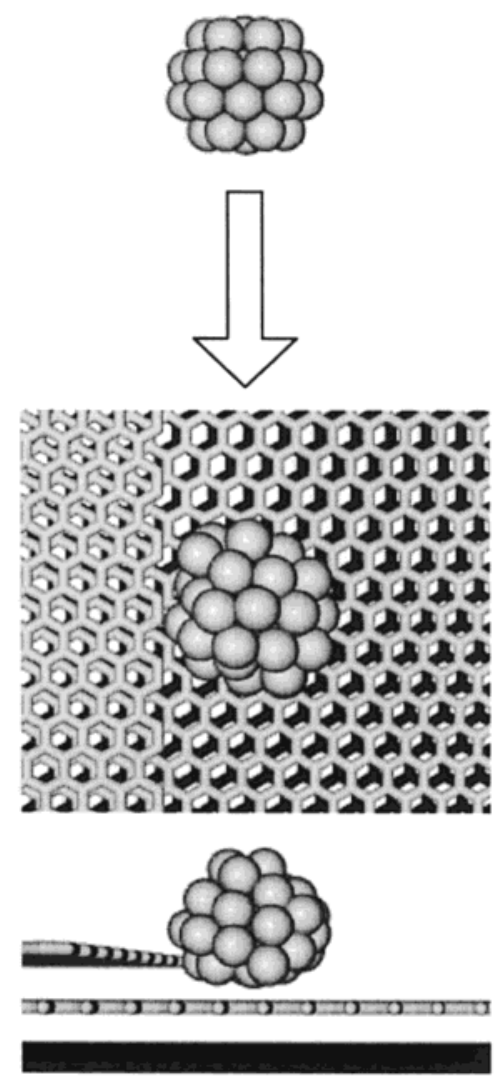

(b)
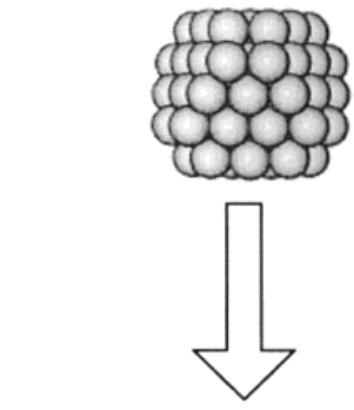

$40+00000$
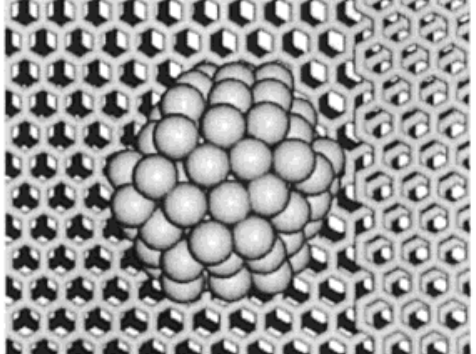

ais
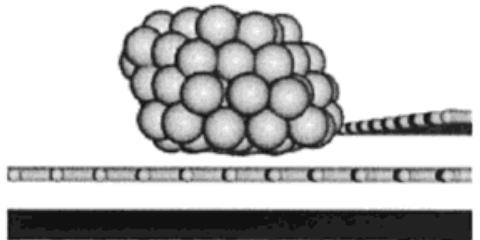

Figure 9. Structural changes of gold clusters during $120 \mathrm{~ns}$ MD simulations: (a) $\mathrm{Au}_{38}$ at $400 \mathrm{~K}$ and (b) $\mathrm{Au}_{79}$ at $500 \mathrm{~K}$. The free clusters are shown in the upper row, top views of the clusters adsorbed on a graphite surface are given in the middle row, and corresponding side views are displayed in the bottom row.

terrace). The $\mathrm{CM}$ trajectory of the $\mathrm{Au}_{140}$ cluster simulated at $300 \mathrm{~K}$ during a $240 \mathrm{~ns}$ time interval, as well as the time development of the $x$ - and $y$-components of the cluster CM, are shown in Figure 12. The CM positions of the cluster, sampled at regular (60 ps) intervals are shown in Figure 13. We observe that the descending steps on both sides act as repulsive walls, and the cluster motion is confined to the terrace. The origin of this cluster-step repulsion is to a large extent the same as in the case of the cluster-hole interaction discussed in section 3.1. When the cluster first begins to overlap the step (starting from the direction of the upper terrace) it loses contact area (and attractive energy) associated with the top terrace, which is replaced by a smaller interaction energy with the more distant lower terrace-this results in an overall energy increase and consequent repulsive forces directed away from the step acting on the cluster that is located on the upper terrace. However, as we discuss below, the maximum energy and repulsive forces occur after the cluster CM has passed slightly over the step and the cluster has begun to tilt so that the above reasoning (i.e., the COM model introduced in section 3.1) does not apply for the entire range as the cluster approaches the energy barrier for going over the step edge. We also remark that in Figure $12 \mathrm{~b}, \mathrm{c}$ there are numerous plateaus in both $X_{\mathrm{CM}}$ and $Y_{\mathrm{CM}}$ (portrayed by the darker regions in Figure 13) that are associated with "stick-slip" diffusive motion" ${ }^{9,10}$ seen both on defect-free surfaces and on the surfaces discussed earlier in this work.

To better understand the nature of the energy and forces associated with the cluster-step interaction, we perform con-
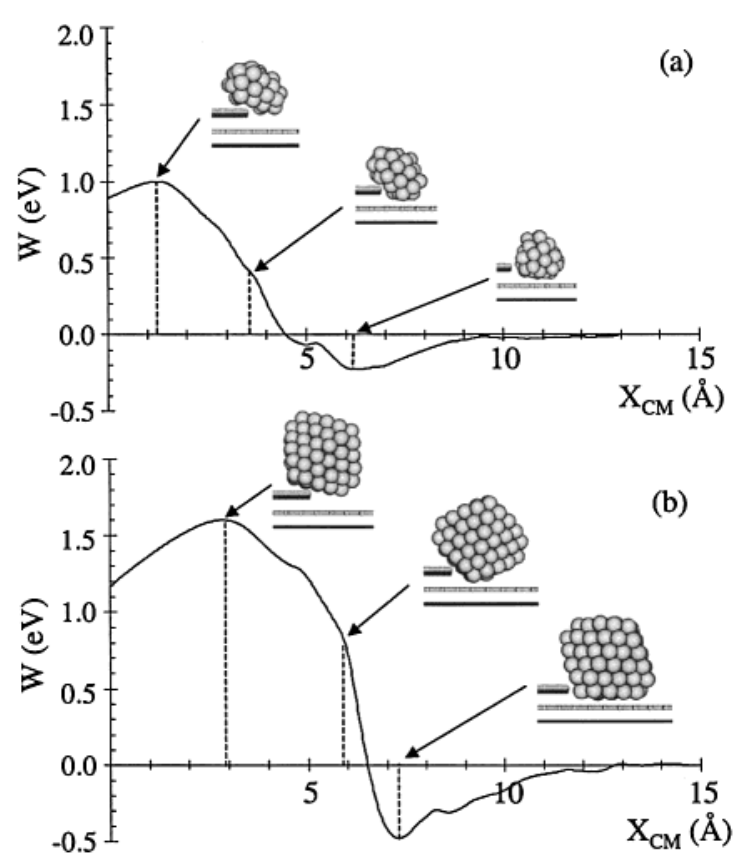

Figure 10. Work of bringing a cluster from a remote location to a distance $X_{\mathrm{CM}}$ from the step edge of a graphite surface, calculated, as described in the text (see eq 6), through the use of constrained MD simulations: (a) $\mathrm{Au}_{38}$ and (b) $\mathrm{Au}_{140}$ at $300 \mathrm{~K}$. Also included are atomistic configurations of the clusters at various stages of the simulations. Note that the positions of the energy minima are slightly larger (by about $1 \AA$ ) than the cluster contact radii. 

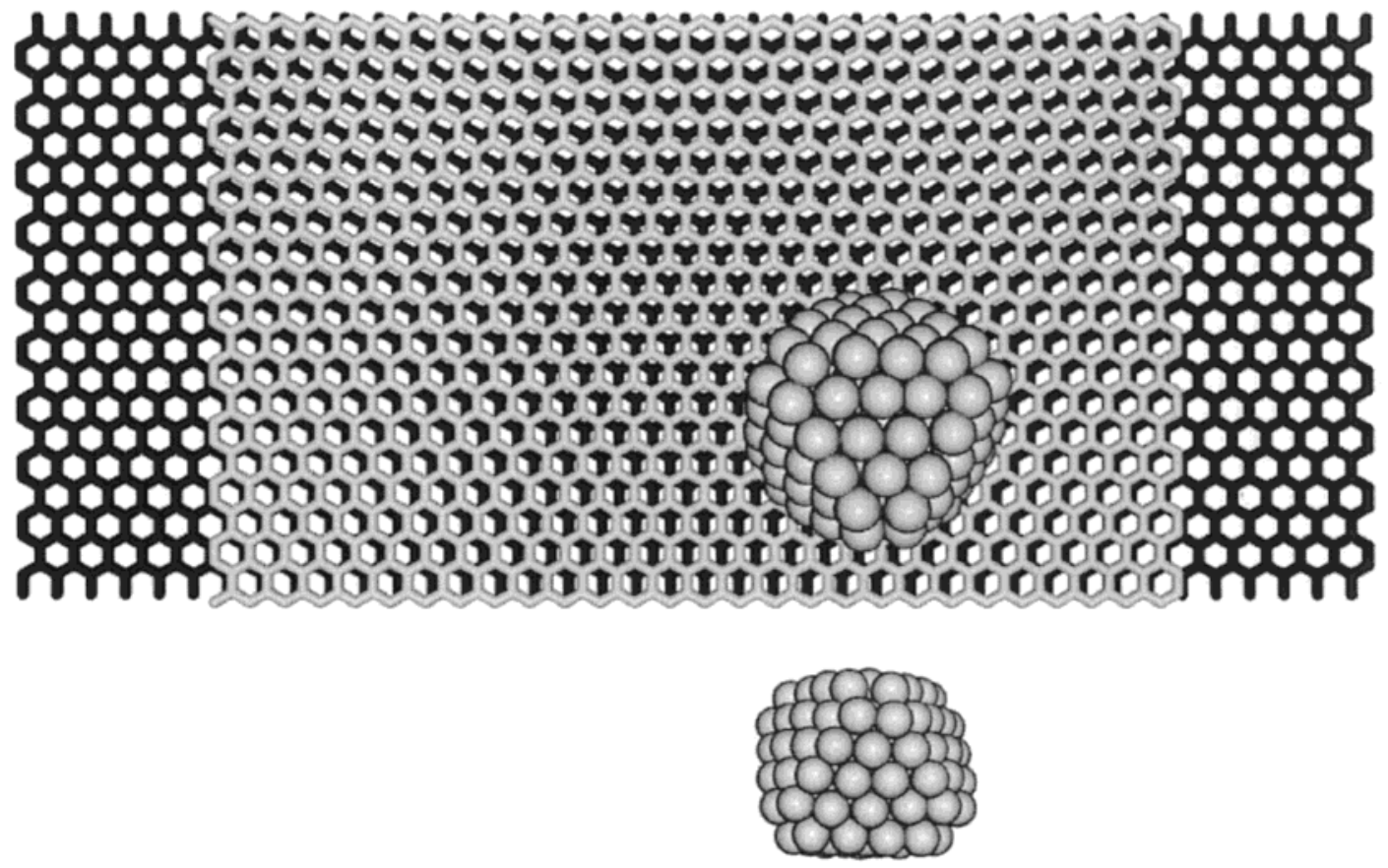

Figure 11. Top view (upper) and side view (bottom) of an $\mathrm{Au}_{140}$ cluster residing on the upper terrace of a graphite surface bounded by two descending steps.
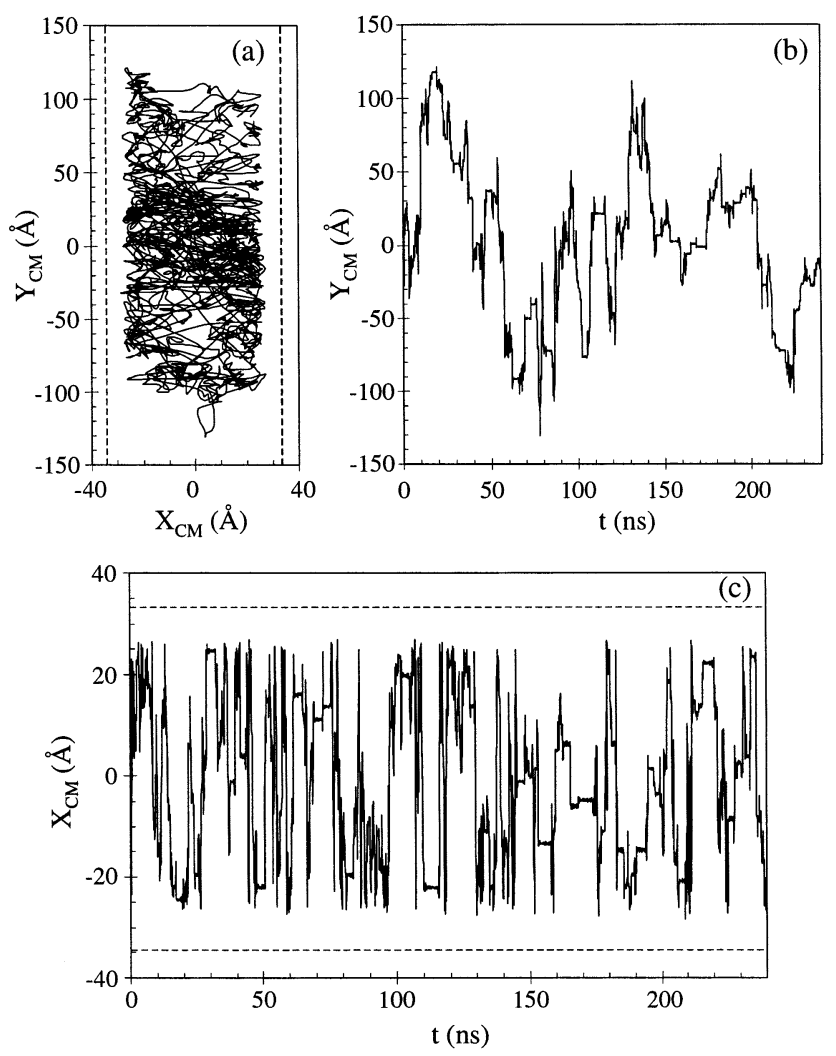

Figure 12. CM trajectories taken from $300 \mathrm{~K}$ MD simulations of an $\mathrm{Au}_{140}$ cluster diffusing on a graphite terrace bounded by two descending steps (dashed lines). Note the excluded zones near the steps (a) and (c) and the presence of plateaus ("stick-slip" diffusion) in (b) and (c).

strained MD simulations for the $\mathrm{Au}_{38}$ and $\mathrm{Au}_{140}$ clusters, similar to those described in the previous section. Taking the step as an origin, $X_{\mathrm{CM}}=0$, we show in Figure 14 the work profiles obtained by integrating the $x$-component of the force on the cluster (see eq 6), along with snapshots of cluster configurations

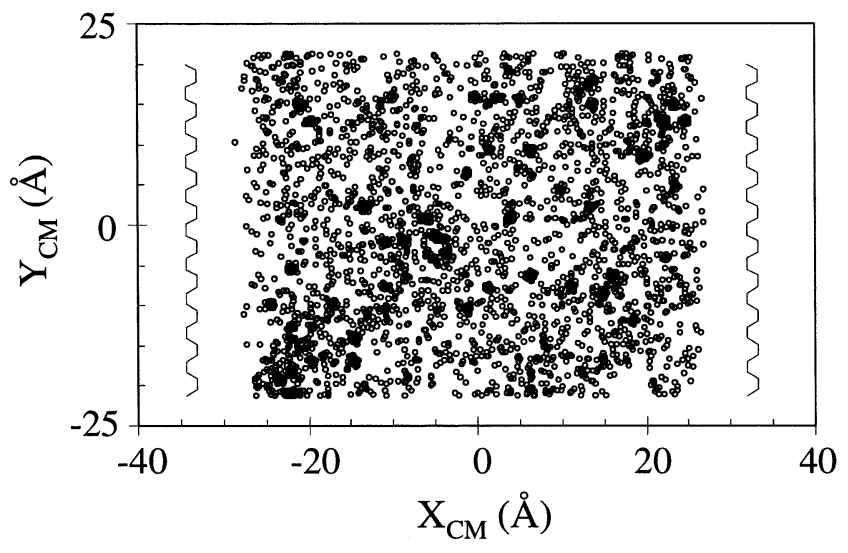

Figure 13. CM positions of the cluster sampled every $60 \mathrm{ps}$ during a $240 \mathrm{~ns}$ MD simulation of an $\mathrm{Au}_{140}$ cluster diffusing on a graphite terrace with linear descending steps. The more darkened regions correspond to the cluster in a vibrational "sticking" state. Note the regions adjacent to the steps in which cluster motion is excluded.

recorded at various stages of the process. The behavior of the clusters again falls into three regimes that are essentially the same as, but in reverse order, to those discussed in section 3.2 for a cluster climbing a step. The important difference between the two cases is that a cluster on a lower terrace is attracted to the step whereas one residing on an upper terrace is repelled from the step, leading in the latter case to an excluded zone near the step edge, as in the cluster-hole case. The width of this excluded zone may be approximated by the radius of the contact area of the cluster with the surface.

It is interesting to note that for the $\mathrm{Au}_{140}$ cluster near an ascending step (see Figures 6 and 10), the longer side of the facetted contact area is located toward the step, thus minimizing the interaction energy; in contrast, for a $\mathrm{Au}_{140}$ cluster approaching a descending step (Figure 14), the longer side (edge of the cluster-surface contact facet) is located away from the step, 

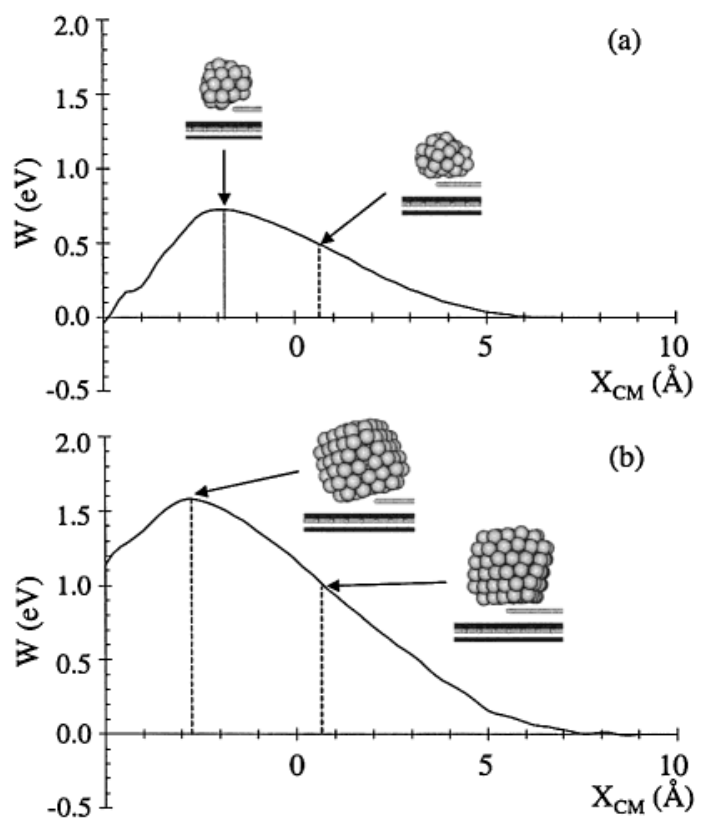

Figure 14. Work required to bring a gold cluster up to, and slightly over, a descending step edge of a graphite surface: (a) $\mathrm{Au}_{38}$ and (b) $\mathrm{Au}_{140}$ at $300 \mathrm{~K}$. Also included are atomic configurations of the cluster during various stages of the constrained MD simulation.

with a consequent minimization of the cluster-step interaction energy.

The energies and forces exerted on a cluster as it approaches a step from the upper terrace side are associated with an effective Schwoebel barrier ${ }^{22}$ for clusters to traverse the step. It is also worth noting that the barrier heights for step traversal are similar for clusters approaching the step from either the ascending or descending side (see Figures 10 and 14). In both cases the energy barrier for traversing the step is $\sim 1.6 \mathrm{eV}$, that is, much larger than an average translational kinetic energy of $\sim 0.04 \mathrm{eV}$ at $300 \mathrm{~K}-$ consequently, one expects step-crossing events to be rare.

As aforementioned (see section 3.2), the development of models for cluster-step interactions that describe the entire steptraversal process are not as straightforward as in the clusterhole case (section 3.1). However, one can make progress in modeling and understanding certain aspects of these interactions. One of the processes that is amenable to a simple model is the approach of a cluster (moving on the upper terrace) to a descending step, as this is similar to a cluster approaching a hole with an effectively infinite radius. Utilizing the same ideas as in the derivation of eqs $2-5$ in section 3.1 , we find the cluster-step interaction energy to be

$$
E=\epsilon A=\epsilon R^{2}\left[\cos ^{-1}\left(\frac{d}{R}\right)-\frac{d}{R} \sqrt{1-\left(\frac{d}{R}\right)^{2}}\right]
$$

and the force $F=-\partial_{d} E$ given by

$$
F=2 \epsilon R \sqrt{1-\left(\frac{d}{R}\right)^{2}}
$$

where $d$ (the argument $d$ in the above equations corresponds to $X_{\mathrm{CM}}$ in the simulations) is the separation between the cluster's $\mathrm{CM}$ and the step (see the diagram in upper right corner of Figure 15) and $\epsilon=\epsilon_{0}-\epsilon_{1}$ as in the cluster-hole interaction. The maximum force is $2 \epsilon R$, and it occurs for $d=0$. Although these results are technically correct for $R>d>-R$, i.e., for the case where a cluster's CM passes over the step, we observe

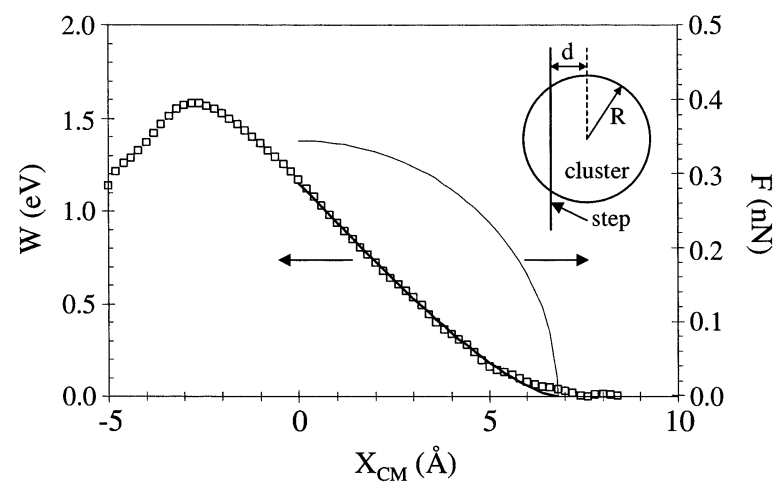

Figure 15. Cluster-step interaction energy of a $\mathrm{Au}_{140}$ cluster starting on the upper terrace of a graphite surface, as it approaches and starts to go over a descending surface step. The energy calculated via the $\mathrm{COM}$ is depicted by the thick solid curve and the energy recorded in the constrained MD simulation is given by the rectangles. The predicted force is also shown (thin solid curve). The cluster approaches the step edge from the right moving on the upper terrace of the step (see insert).

that for the weak cluster-graphite interactions operative in this study, the cluster begins to tilt as its CM approaches the step edge $(d=0$; see also Figure 14). Additionally, the maximum in the cluster-step interaction (the step barrier) occurs after the cluster CM has passed beyond the step edge. Consequently, the cluster-overlap ideas, which assume full cluster-surface contact (i.e., no tilting), are applicable approximately in the range $R>d>0$.

For $\mathrm{Au}_{140}$, we have compared the model to the simulation results by applying a nonlinear least-squares fit with $R$ and $\epsilon$ as fitting parameters over the range $R>d>0$. We obtain $R=$ $6.82 \AA$ and $\epsilon=0.158 \mathrm{eV} / \AA^{2}$, which are merely $8 \%$ and $14 \%$ different from the values obtained for the $\mathrm{Au}_{140}$-hole interaction earlier using the COM model (section 3.1) Consequently, within its range of applicability, i.e., when the cluster's CM is on the upper terrace and tilting is insignificant, this model gives reasonable results. ${ }^{23}$ The energy calculated from this model at $d=0$ (see Figure 15) is $\sim 1.1 \mathrm{eV}$ compared to the full energy barrier of $\sim 1.6 \mathrm{eV}$. The mean translational kinetic energies for clusters over a wide temperature range are much lower than the above value (e.g., for temperatures between 300 and 1000 $\mathrm{K}$, the mean kinetic energies are $\sim 0.04-0.13 \mathrm{eV}$ ) - this suggests that eqs 7 and 8 may be utilized in certain MD and Monte Carlo simulations.

\section{Summary}

In this study we investigated with the use of extensive molecular dynamics simulations the energetics and diffusion characteristics of gold clusters (consisting of 38, 79, 140, or 586 atoms) on two types of defective graphite surfaces: (1) in section 3.1 we considered a one-layer deep hole (consisting of a 13-atom extended vacancy in the topmost layer), and (2) in sections 3.2 and 3.3 we discussed graphite surfaces with linear (straight) one-layer high steps, with the adsorbed cluster starting on the bottom (section 3.2) or top (section (3.3) terrace of the stepped surface.

For the extended hole defect we found that the hole acts as a repulsive barrier to the diffusing clusters, resulting in an excluded zone around the hole with a radius close to the sum of the cluster and hole radii. Similarly, a cluster diffusing on a top terrace of a stepped graphite surface is repelled away (via a Schwoebel-like barrier) from the step edge connecting to a lower terrace. On the other hand, the diffusion of a cluster starting on a bottom terrace of a stepped surface is constrained 
by the ascending step edges to remain on that terrace; at low temperatures the cluster is trapped to a region of the lower terrace in the vicinity of the step edge (diffusing along the step edge), and at higher temperature it may escape from the stepedge region and diffuse on various regions of the bottom terrace. The calculated values of the energy barriers for traversing the hole defect (or entering the excluded zone) and the aforementioned Schwoebel-like barrier for motion over a descending step edge, are much larger then the center-of-mass kinetic energy of the clusters at room temperature, and consequently such barrier crossing events are exceedingly rare. Similarly, the calculated depth of the trapping potential near the ascending step edge (for a cluster approaching the step edge from the bottom graphite layer) is large enough to limit the motion of the cluster to the vicinity of the step edge, and the barrier for climbing the step is high enough to restrict the motion of the cluster to the lower graphite layer.

The simulations guided formulation of a simple analytic phenomenological model (the cluster overlap model, COM) that has been demonstrated (see sections 3.1 and 3.3) to provide an appropriate description of the energy and of the forces acting on a cluster diffusing on a graphite surface in the presence of defects.

These simulations provide valuable information of relevance to investigations of thin film growth processes using cluster deposition $^{1-6}$ as well as for the development of surface patterning methods. For example: (i) trapping of clusters at the step edges of ascending steps may be used for generation of quasi-one-dimensional "cluster wires", (ii) a surface patterned by hole defects may serve as a template for generation of thin films with a hole (extended vacancy) pattern ("anti-dots"), and (iii) clusters trapped in large surface holes (i.e., craters) may serve as nucleation centers for patterned growth of surface films.

Acknowledgment. This work was supported by the US Department of Energy Grant No. FG05-86ER-45234. The simulations were performed at the Georgia Institute of Technology Center for Computational Materials Science.

\section{References and Notes}

(1) NATO-ASI Surface Diffusion: Atomistic and Collective Processes; Tringides, M. C., Ed.; Plenum: New York, 1997. Wang, S. C.; Kurpick, U.; Ehrlich, G. Phys. Rev. Lett. 1998, 81, 4923 and references therein.
(2) Nanoparticles and Nanostructured Films; Fendler, J. H., Ed.; WileyVCH: Weinheim, 1998.

(3) Jensen, P. Rev. Mod. Phys. 1999, 71, 1695.

(4) Bardotti, L.; Jensen, P.; Hoareau, A.; Treilleux, M.; Cabaud, B.; Perez, A.; Cadete Santos Aires, F. Surf. Sci. 1996, 367, 276.

(5) Yoon, B.; Akulin, V.; Cahuzac, P.; Carlier, F.; de Frutos, M.; Masson, A.; Mory, C.; Colliex, C.; Bréchignac, C. Surf. Sci. 1999, 443, 76.

(6) Bréchignac, C.; Cahuzac, P.; Carlier, F.; Colliex, C.; Leroux, J.; Masson, A.; Yoon, B.; Landman, U. Phys. Rev. Lett. 2002, 88, 196103.

(7) (a) Stolt, K.; Graham, W. R.; Ehrlich, G. J. Chem. Phys. 1976, 65, 3206. (b) Landman, U.; Montroll, E. W.; Shlesinger, M. F. Phys. Rev. Lett. 1977, 38, 285, and references to early experiments cited therein.

(8) Hamilton, J. C.; Daw, M. C.; Foiles, S. M. Phys. Rev. Lett. 1995, 74, 2760, Landman, U.; Luedtke, W. D. Appl. Surf. Sci. 1996, 92, 237.

(9) Luedtke, W. D.; Landman, U. J. Phys. Chem. 1996, 100, 13323.

(10) Luedtke, W. D.; Landman, U. Phys. Rev. Lett. 1999, 82, 3835; 83, 1702 (erratum).

(11) Lévy Flights and Related Topics in Physics; Shlesinger, M. F., Zaslavsky, G. M., Frisch, U., Eds.; Springer: Berlin, 1995.

(12) Deltour, P.; Barrat, J.-L.; Jensen, P. Phys. Rev. Lett. 1997, 78, 4597.

(13) Noll, J. D.; Copper, J. B.; Myrick, M. L. J. Vac. Sci. Technol. B 1993, 11, 2006

(14) Rong, Z. Y. Phys. Rev. B 1994, 50, 1839.

(15) Hahn, J. R.; Kang, H. Phys. Rev. B 1999, 60, 6007.

(16) An, B.; Fukuyama, S.; Yokogawa, K.; Yoshimura, M. J. Appl. Phys. 2002, 92, 2317

(17) Adams, J. B.; Foiles, S. M.; Wolfer, W. G. J. Mater. Res. 1989, 4, 02.

(18) Yoon, B.; Luedtke, W. D.; Landman, U. To be published, 2003.

(19) Indeed, in light of the coexistence of long flights and sticking events found in the above simulations, we note that several studies, pertaining to the connection between Lévy-type motion and the character of the ensuing diffusion (that is, the exponent $\gamma$ ), have shown (see refs 20 and 21) that the interplay between the flight and sticking exponents ( $\mu$ and $v$, respectively) can lead to crossovers between the various diffusion regimes (i.e., regular with $\gamma=1$ and anomalous with $\gamma \neq 1$ ).

(20) Klafter, J.; Zumofen, G.; Shlesinger, M. F. In Lévy Flights and Related Topics in Physics; Shlesinger, M. F., Zaslavsky, G. M., Frisch, U., Eds.; Springer: Berlin, 1995; p 196.

(21) Zaslavsky, G. M. In Lévy Flights and Related Topics in Physics; Shlesinger, M. F., Zaslavsky, G. M., Frisch, U., Eds.; Springer: Berlin, 1995 p 216.

(22) Schwoebel, R. L.; Shipsey, E. J. J. Appl. Phys. 1966, 37, 3682.

(23) We note that all of the energies and forces for the models presented here scale with the effective interaction energy $\epsilon=\epsilon_{0}-\epsilon_{1}$. For a study such as this, the $\mathrm{Au}-$ graphite energies are best estimates. If one wants to apply these models with a more precise estimate of the interaction energies and energy barrier, then $\epsilon$ may be used as a fitting parameter. 